\title{
Design of automated driving system of a wheeled heavy duty off-road vehicle
}

\author{
Xiaoyun Li, Guangming Xiong, Min Zhu and Yu Zhang \\ Intelligent Vehicle Research Center, Beijing Institute of Technology, Beijing 100081, China \\ Ixy900725@163.com
}

Keywords: wheeled heavy duty off-road vehicle; automated driving system; lateral control; longitudinal control.

Abstract. Automatic control system is the guarantee for the autonomous system to realize automation. In this paper, an automatic control system including lateral control, longitudinal control and integrated control based on x-by-wire is proposed for a wheeled heavy duty off-road vehicle. The experimental results show the effectiveness of the proposed system.

\section{Introduction}

In January 2015, Mercedes-Benz introduced a fully autonomous concept called F 015 Luxury in Motion at the Consumer Electronics Show (CES) in Las Vegas, Nevada [1], showing its advanced technology in automated driving and enormous ambition in this field. Coincidentally, General Motors, BMW, Audi, etc. have also planned to launch their own intelligent vehicles featured in automated driving in 2015-2018 [2, 3]. It is obvious that the intelligent vehicle technology has aroused great interest in automotive field and developed rapidly to an advanced stage in recent years [4].

Although research on autonomous vehicles is frequently reported nowadays, it mainly focusses on small vehicles. There is little research on the wheeled heavy duty off-road autonomous vehicles. Team Oshkosh's vehicle, TerraMax, for example, is a typical wheeled heavy duty off-road autonomous vehicle kit system that advances an automatic control system to increase performance in autonomous missions $[5,6,7,8]$. In this paper, concentrated on a wheeled heavy duty off-road vehicle, we realize its modification by designing an automated driving system consisting of lateral control, longitudinal control and integrated control based on x-by-wire, so as to achieve automated driving in real scenarios.

\section{Design of the Lateral Control System}

Lateral control system is also called automatic steering control system. Because of the complexity of vehicle structure and the variability of vehicle-road interaction, lateral control becomes quite difficult, which draws higher requests on the control of the autonomous vehicle. Especially the modeling of the wheeled autonomous vehicle's lateral control system, the whole system's input and output response, as well as the settings of feedback, all of which will finally affect the accuracy of lateral control. Lateral control system leads the autonomous vehicle to drive along the desired path through the reasonable control of steering angle, so as to ensure riding comfort and handling stability. The overall lateral control framework is shown in Fig. 1.

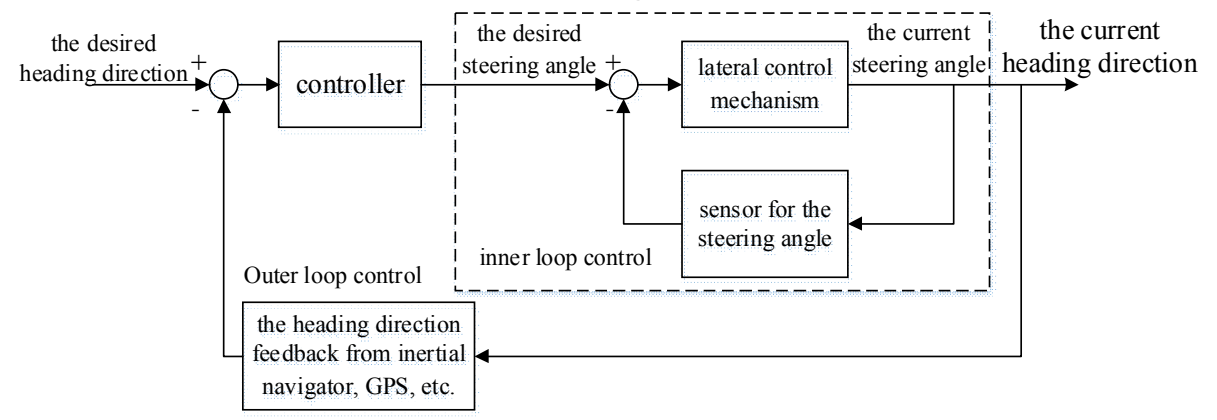

Fig. 1 The overall lateral control framework 
As shown in Fig. 1, the lateral control system consists of two control loops, i.e., inner loop and outer loop. Based on the deviation of the desired steering angle and the current steering angle, the inner loop can realize precision control of steering angle by actuating the lateral control mechanism. Based on the deviation of the desired heading direction and heading direction feedback from inertial navigator, GPS, etc., the outer loop can calculate the desired steering angle by automatic steering control algorithm.

\section{Design of the Longitudinal Control System}

Longitudinal control system mainly includes shift control, engine control and brake control, and its control objective is to track the speed given by the upper computer.

Design of the Shift Control System. Shift control requests controlling automatic transmission and torque converter with less modification. In order to use the shift function both in manual and automated driving states, the system applies an additional controller to receive signals from the original handle or the upper computer, then sends shift orders by the additional controller to the transmission controller, so as to realize the shift process.

Design of the Engine Control System. The engine applied in the automatic control platform is a cummins diesel engine. The rotation speed control can be realized in three ways: (a) Simulating the voltage signals of throttle opening by ECU; (b) Simulating the digital signals of CAN communications throttle opening; (c) Requesting engine speed adjustment directly by CAN communications. Since (a) needs to add the extra analog circuit in the controller, and the rotation speed control in (a) and (b) is indirect with nonlinearity introduced, (c) is applied as our final approach to realize the rotation speed control, which functions as requesting rotation speed and converting it to velocity. Compared with the other two approaches, (c) is more intuitive, accurate and stable with less modification in hardware.

Design of the Brake Control System. Brake control requests the autonomous vehicle to realize service brake and parking brake, as well as switching to the original manual driving mode with no apparent influence on brake performance.

The brake control system adds two valve banks of active service brake and one valve bank of active parking brake. The control part adds a brake controller. The valve banks of active service brake can adjust the brake pressure proportionally, while the valve bank of active parking brake functions as switching adjustment. The brake controller can realize active service brake and parking brake based on the collected inputs and inputs from CAN.

\section{Experimental Results}

Experimental Results of Automatic Steering. The lateral tracking control experiment based on the classic PID control algorithm is conducted. After multiple debugging, the optimal parameters are obtained, i.e., $K_{\mathrm{P}}=120, K_{\mathrm{I}}=0, K_{\mathrm{D}}=0$. And four experiments are conducted under the condition of $0^{\circ} \sim 15^{\circ}, 340^{\circ} \sim 315^{\circ}, 50^{\circ} \sim 315^{\circ}$ and $350^{\circ} \sim 260^{\circ}$, respectively.

Experimental Results of Electronic Brake. Fig. 2 illustrates the process of an electronic brake experiment on the off-road. Lots of experiments show that the modified electronic brake system of the vehicle can fulfill brake function in the state of automated driving. 


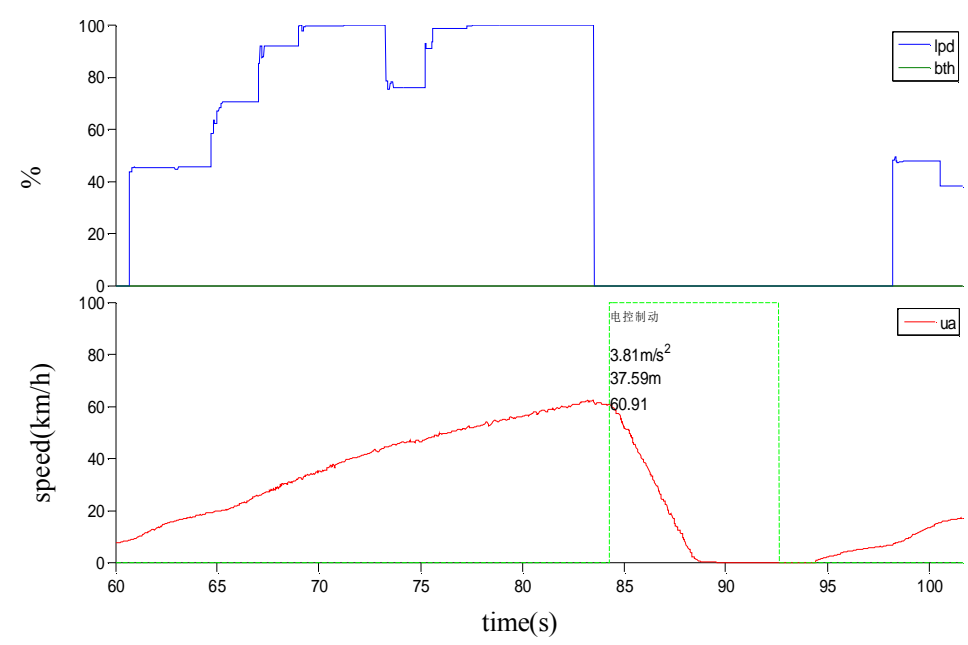

Fig. 2 The process of electronic brake on the off-road

Experimental Results of Speed Control. Fig. 3 shows that in the process of acceleration, PID controller is used to drive the vehicle with a large acceleration at first, then the acceleration begins to decrease as the current speed gets close to the desired speed. Although overshoot exists to some extent, the speed converges quickly to $10 \mathrm{~m} / \mathrm{s}$ and the vehicle will drive at the constant speed of $10 \mathrm{~m} / \mathrm{s}$ stably. Moreover, the process of deceleration also accords with the characteristics of drivers, and the vehicle can quickly slow down to $0 \mathrm{~m} / \mathrm{s}$ so as to shorten the braking distance. To achieve the effect of a slow braking, the deceleration begins to decrease as the current speed gets close to $0 \mathrm{~m} / \mathrm{s}$. Fig. 3 shows the control result of speeds $0-5-7-10-6-0 \mathrm{~m} / \mathrm{s}$. The vehicle's initial speed is $0 \mathrm{~m} / \mathrm{s}$, then the desired speed of $5 \mathrm{~m} / \mathrm{s}$ is sent to the PID controller in 10 seconds, then $7 \mathrm{~m} / \mathrm{s}, 10 \mathrm{~m} / \mathrm{s}$ and $6 \mathrm{~m} / \mathrm{s}$ are sent in turn in a period, and finally $0 \mathrm{~m} / \mathrm{s}$ to brake. During the automated driving, the self-tuning system of PID controller adjusts $K_{\mathrm{P}}, K_{\mathrm{I}}$ and $K_{\mathrm{D}}$ in real time based on the deviation of vehicle's desired speed and current speed, as well as the gradient of the deviation. In this way, PID controller succeeds in realizing the quick and stable response of the vehicle. In Fig. 3, it can be found that the process of speed decreasing from $10 \mathrm{~m} / \mathrm{s}$ to $6 \mathrm{~m} / \mathrm{s}$ exists large overshoot, since the vehicle is performing an automatic shift. But the self-tuning system can adjust the parameters, making the speed converge quickly to $6 \mathrm{~m} / \mathrm{s}$. Overall, the results indicate that the incremental self-tuning PID algorithm shows fast response, high precision and good stability in the vehicle's speed control.

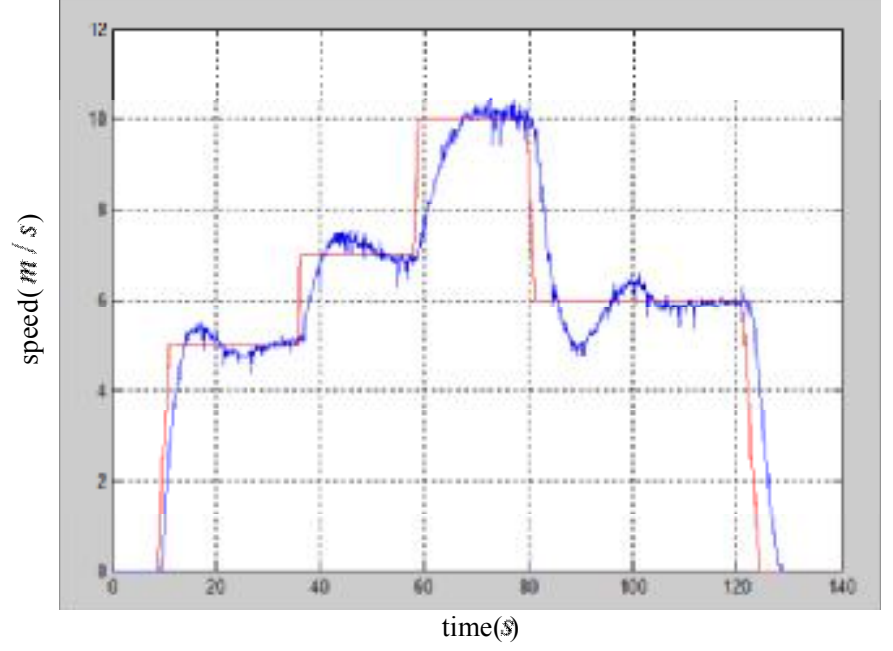

Fig. 3 The experimental results of the incremental self-tuning PID algorithm

\section{Conclusion}

In this paper, we proposed an automatic control system of a wheeled heavy duty off-road autonomous vehicle. Consisting of lateral control, longitudinal control and integrated control based on 
x-by-wire, the system can realize automatic steering control, speed control and other actuators control, thus achieve automated driving effectively. Experimental results show that the proposed system and algorithm are efficient and can be used for the wheeled heavy duty off-road autonomous vehicle to achieve automated driving in real scenarios.

\section{References}

[1] Information on

http://www.leftlanenews.com/mercedes-benz-f-015-luxury-in-motion.html\#ixzz3PMJOTnf3

[2] Information on

http://www.worldcarfans.com/111062334412/volkswagens-temporary-auto-pilot-makes-for-a-self-d riving

[3] Information on

http://www.slate.com/articles/technology/future_tense/2011/07/life_is_a_selfdriving_highway.html

[4] J. y. Lian, The Intelligent vehicle control system Based on the fuzzy neural network technology, Advanced Materials Research. 487(2012) 830-835.

[5] Information on http://oshkoshdefense.com/technology-1/unmanned-ground-vehicle/

[6] A. Broggi, A. Cappalunga, C. Caraffi, S. Cattani, S. Ghidoni, P. Grisleri, P. P. Porta, M. Posterli, P. Zani, J. Beck, The passive sensing suite of the TerraMax autonomous vehicle, 2008 IEEE Intelligent Vehicles Symposium. 769-774.

[7] D. Braid, A. Broggi, G. Schmiedel, The TerraMax autonomous vehicle, Journal of Field Robotics. 23(9) 693-708.

[8] U. Ozguner, K. A. Redmill, A. Broggi, Team TerraMax and the DARPA grand challenge: a general overview, 2004 IEEE Intelligent Vehicles Symposium. 232-237. 